\title{
Valores de referência de parâmetros bioquímicos e hematológicos de Rattus novergicus (Wistar) da Unidade de Produção Animal do Instituto de Pesquisa em Fármacos e Medicamentos da Universidade Federal da Paraíba
}

\author{
Reference values of biochemical and hematological parameters of Rattus novergicus (Wistar) \\ from the Animal Production Unit of the Research Institute for Drugs and Medicines of the \\ Federal University of Paraíba
}

\author{
F. A. S. Oliveira'; R. C. Ferreira ${ }^{2}$; R. N. Parentoni ${ }^{3}$; C. C. N. Andrade ${ }^{2}$; A. L. O. \\ Lopes $^{2}$; A. L. G. Cruz ; S. S. Duarte'; D. K. F. Silva²; V. M. de Sousa²; T. M. \\ Batista $^{2}$; I. B. S. Gomes ${ }^{5}$; M. V. Sobral ${ }^{2 *}$ \\ ${ }^{1}$ Programa de Pós-graduação em Ciências Farmacêuticas, Universidade Federal de Pernambuco, 50670-901, Recife- \\ Pernambuco, Brasil \\ ${ }^{2}$ Programa de Pós-graduação em Produtos Naturais e Sintéticos Bioativos, Universidade Federal da Paraíba, 58051- \\ 900, João Pessoa-Paraíba, Brasil \\ ${ }^{3}$ Instituto de Pesquisa em Fármacos e Medicamentos, Universidade Federal da Paraíba, 58051-900, João Pessoa- \\ Paraíba, Brasil \\ ${ }^{4}$ Centro de Ciências Exatas e da Natureza, Universidade Federal da Paraíba, 58051-900, João Pessoa-Paraíba, Brasil \\ ${ }^{5}$ Laboratório de Bioquímica Clínica, Departamento de Ciências Farmacêuticas, Universidade Federal da Paraíba, \\ 58051-900, João Pessoa-Paraíba, Brasil \\ *mariannavbs@gmail.com \\ (Recebido em 01 de outubro de 2020; aceito em 22 de março de 2021)
}

\begin{abstract}
Animais de diferentes criadouros podem apresentar variações em seus parâmetros fisiológicos. Os objetivos desse trabalho foram determinar o intervalo de valores de referência de parâmetros bioquímicos e hematológicos de ratos e ratas Wistar (Rattus norvegicus) da Unidade de Produção Animal do Instituto de Pesquisa em Fármacos e Medicamentos da Universidade Federal da Paraíba (UPA/IPeFarM/UFPB), bem como comparar os dados encontrados com dados publicados para outras unidades de produção animal nacionais e internacionais. Foram utilizados animais saudáveis ( $\mathrm{n}=40 /$ gênero), com 8 a 12 semanas de idade e peso entre 150 e $300 \mathrm{~g}$. Amostras de sangue total foram obtidas por punção intracardíaca e utilizadas para a quantificação de eritrócitos, leucócitos, plaquetas e dos índices hematimétricos. Foram determinadas as concentrações séricas de glicose, triglicérides, colesterol, proteínas totais, ureia, albumina, creatinina, ácido úrico e a atividade enzimática da aspartato aminotransferase, alanina aminotransferase e fosfatase alcalina. Ao comparar os gêneros, foram observadas diferenças significativas entre todos os parâmetros avaliados. Além disso, foram encontradas discrepâncias entre os valores obtidos (exceto proteínas totais, ácido úrico e hemácias) e resultados de outros criadouros. É necessário, portanto, que cada biotério determine os valores de referência do perfil fisiológico de seus animais, considerando os fatores intrínsecos e extrínsecos na homeostase dos mesmos, a fim de fornecer dados reais para os experimentos de suas instituições.

Palavras-chave: ratos Wistar, bioquímica, hematologia.
\end{abstract}

Animals from different breeding sites may show variations in their physiological parameters. The aims of this study were to determine the range of reference values for biochemical and hematological parameters of Wistar rats (Rattus norvegicus) from the Animal Production Unit of the Research Institute for Drugs and Medicines at the Federal University of Paraíba (UPA/IPeFarM/UFPB), as well as comparing the data found with published data for other national and international animal production units. Healthy animals $(\mathrm{n}=40$ /gender) with 8 to 12 weeks of age and weight between 150 and $300 \mathrm{~g}$ were used. Whole blood samples were obtained by intracardiac puncture and used for the quantification of erythrocytes, leukocytes, platelets and hematimetric indices. Serum concentrations of glucose, triglycerides, cholesterol, total proteins, urea, albumin, creatinine, uric acid and the enzymatic activity of aspartate aminotransferase, alanine aminotransferase and alkaline phosphatase were determined. When comparing the genders, significant differences were observed between all parameters evaluated. In addition, discrepancies were found between the values obtained (except total proteins, uric acid and red blood cells) and results from other breeding sites. Therefore, it is necessary that each bioterium determines the reference values of the physiological profile of 
their animals, considering the intrinsic and extrinsic factors in their homeostasis, in order to provide real data for the experiments of their institutions.

Keywords: Wistar rats, biochemistry, hematology.

\section{INTRODUÇÃO}

O emprego de animais na investigação científica é uma prática milenar, que se originou com os estudos de Hipócrates e se estende até os dias atuais, constituindo uma das principais fontes para obtenção de dados biológicos. A elucidação dos processos vitais, bem como as descobertas da insulina, das vacinas, dos medicamentos, dos métodos de prevenção e diagnóstico de doenças, e transplante de órgãos são alguns dos vários avanços adquiridos com as pesquisas in vivo que mudaram o rumo da medicina humana e veterinária $[1,2,3]$.

Em virtude das discussões éticas a respeito dos estudos in vivo e da facilidade de aquisição, manejo, adaptação, manutenção e observação, as espécies mais intensamente utilizadas e conhecidas no meio científico são: o camundongo (Mus musculus), o rato (Rattus novergicus), o hamster (Mesocretus auratus), a cobaia (Cavia porcellus) e o coelho (Oryctolagus cuniculus) [1, $4,5]$. O rato foi um dos primeiros animais a ser domesticado para fins estritamente científicos, com estimativa de que seu emprego represente cerca de $30 \%$ do número total de animais utilizados em laboratório [6,7]. Dessa espécie, a linhagem Wistar, albina e heterogênea, é uma das mais empregadas nos centros de pesquisa, tendo grande aplicação no estudo de doenças poligênicas, como o diabetes mellitus, bem como na oncologia e em estudos relacionados a nutrição e envelhecimento $[8,9]$.

Os animais experimentais possuem necessidades metabólicas, energéticas e nutricionais que variam de acordo com a região do planeta e com as condições as quais estão submetidos. Isso faz com que diferenças relevantes sejam verificadas nos parâmetros bioquímicos e hematológicos, por exemplo, em animais de mesma espécie e linhagem, sob condições de acondicionamento diferentes [10]. Em decorrência disso, variáveis relacionadas com o ambiente, como temperatura, umidade, iluminação e ruído, devem ser padronizadas, dentro dos limites aceitáveis, no intuito de garantir o bem-estar animal e reduzir as diferenças nos parâmetros fisiológicos, em estudos in vivo. Essas têm sido uma prioridade de países com larga tradição em biotérios, os quais determinam os valores de referência dos parâmetros bioquímicos e hematológicos dos animais utilizados em suas instituições de pesquisa, considerando as variáveis intrínsecas e extrínsecas aos mesmos $[7,11,12]$.

A World Health Organization (WHO), International Federation of Clinical Chemistry and Laboratory Medicine (IFCC) e o Clinical and Laboratory Standards Institute (CLSI) definem "valor de referência" como o resultado obtido da observação ou mensuração quantitativa de um analito em um indivíduo/animal selecionado, com base em critérios bem definidos. Os valores de referência dos perfis bioquímico e hematológico determinam o número de células e as taxas dos componentes não celulares do sangue, considerados normais de uma determinada população. Esses dados têm orientado as pesquisas farmacológicas e toxicológicas, no intuito de fornecer informações que subsidiam a relação entre a análise do sangue de animais sadios, doentes e tratados com amostras em estudo, contribuindo para uma discussão mais coerente e fiel dos resultados [3, 7].

No Brasil, poucos laboratórios têm se dedicado a estabelecer seus próprios intervalos fisiológicos, quando se utiliza plasma/soro de animais. Alternativamente, as faixas de valores utilizadas são provenientes da literatura estrangeira, oriunda de países que mantêm um controle rigoroso de seus biotérios, ou dos dados que constam nas instruções de uso dos fabricantes dos reagentes $[13,14]$. Contudo, esses valores não são fidedignos a realidade brasileira, uma vez que, apesar de corresponderem a mesma espécie e linhagem, os animais podem expressar diferentes perfis bioquímico e hematológico [10].

Portanto, é imprescindível que cada instituição de pesquisa atualize, periodicamente, os parâmetros fisiológicos considerados normais dos animais que são utilizados na investigação científica. Diante disso, o presente trabalho teve como objetivos atualizar a faixa de valores de referência de parâmetros bioquímicos e hematológicos dos ratos Wistar (Rattus norvegicus) provenientes da Unidade de Produção Animal (UPA) do Instituto de Pesquisa em Fármacos e Medicamentos (IPeFarM) da Universidade Federal da Paraíba (UFPB), bem como comparar os 
dados encontrados com dados publicados para outras unidades de produção animal nacionais e internacionais.

\section{MATERIAL E MÉTODOS}

Foram utilizados ratos e ratas Wistar (Rattus norvegicus), 40 animais por gênero, selecionados de ninhadas diferentes, pesando entre 150 e $300 \mathrm{~g}$, com faixa etária entre 8 e 12 semanas, independente do ciclo estral. Os mesmos foram agrupados em gaiolas de polietileno, sob condições controladas de temperatura $\left(21 \pm 1{ }^{\circ} \mathrm{C}\right)$, com livre acesso à comida (pellets de ração Presence $\left.{ }^{\circledR}\right) \mathrm{e}$ água potável, e foram mantidos em ciclo claro-escuro de doze horas (luzes acesas às 6:00 da manhã). Os procedimentos experimentais foram aprovados pela Comissão de Ética no Uso de Animais (CEUA/UFPB), sob a certidão $n^{\circ} 2895270218$.

Após jejum alimentar de doze horas, os animais foram submetidos a injeção intraperitoneal (i.p.) de xilazina $(30 \mathrm{mg} / \mathrm{kg}$ ) e cetamina $(300 \mathrm{mg} / \mathrm{kg}$, i.p.), para coleta de sangue por via intracardíaca, seguido de eutanásia.

Para a análise dos parâmetros bioquímicos (glicose, triglicérides, colesterol total, aspartato aminotransferase (AST), alanina aminotransferase (ALT), fosfatase alcalina (FAL), proteínas totais, ureia, albumina, creatinina e ácido úrico), o sangue foi submetido à centrifugação (10 minutos, a $3500 \mathrm{rpm}$ ), em tubos com gel separador para obtenção do soro. Já para as análises hematológicas (contagem de eritrócitos, largura de distribuição dos eritrócitos (RDW), hemoglobina, hematócrito (HCT), volume corpuscular médio (VCM), hemoglobina corpuscular média (HCM), concentração da hemoglobina corpuscular média (CHCM), contagem de leucócitos, neutrófilos, linfócitos, monócitos, eosinófilos e plaquetas, volume plaquetário médio (VPM), largura de distribuição das plaquetas (PDW), foi utilizado sangue total heparinizado.

Os parâmetros bioquímicos e hematológicos foram determinados utilizando-se kits específicos $\left(\right.$ Bioclin $^{\circledR}$ ) para o espectrofotômetro 600 plus $\left(\right.$ FEMTO $^{\circledR}$ ) e UV mini-1240 (UV-VIS SPECTROPHOTOMERS, SHIMADZU ${ }^{\circledR}$ ), e para o analisador hematológico celular automático Hematoclin 2.8 Vet (Bioclin/Midray $\left.{ }^{\circledR}\right)$, respectivamente. As extensões sanguíneas foram coradas com coloração panótica para contagem diferencial de leucócitos em microscópio óptico $\left(\right.$ OLYMPUS $\left.^{\circledR}\right)$.

Os resultados foram expressos como média aritmética \pm desvio padrão (DP) da média e analisada pelo teste de análise de variância (ANOVA) one-way, seguido do teste de Tukey. As diferenças entre os grupos foram consideradas significativas quando apresentaram $p<0,05$.

Para determinação dos intervalos de referência, foram estabelecidos os limites inferior e superior como sendo a média aritmética $\pm 2 \mathrm{DP}$, o que abrange $95 \%$ dos dados centrais da curva de distribuição Gaussiana, com uma margem de erro em $5 \%$ dos casos.

\section{RESULTADOS E DISCUSSÃO}

Os parâmetros hematológicos e bioquímicos são amplamente utilizados como indicadores fisiológicos dos animais em resposta a alterações endógenas e como biomarcadores de diagnóstico. Alterações nesses parâmetros podem sugerir, por exemplo, lesões em órgãos ou tecidos específicos, auxiliar na avaliação toxicológica de candidatos a fármacos e na detecção de deficiências do sistema de criação das espécies [15]. Os valores de referência desses parâmetros, quando corretamente interpretados, podem demonstrar, portanto, as condições do animal no momento da amostragem [16].

A construção dos valores de referência, no entanto, pode se tornar uma tarefa dispendiosa e exaustiva para os laboratórios [14], pois, segundo a International Federation of Clinical Chemistry (IFCC) e a American Society for Veterinary Clinical Pathology (ASVCP), a quantidade ideal de amostras necessárias seria acima de 120 animais saudáveis por gênero, idade, espécie e linhagem. Por esse motivo, tais órgãos permitem caracterizações preliminares dos animais de laboratório com um número inferior, as quais devem ser renovadas periodicamente $[17,18]$. No presente estudo foram determinadas as faixas de valores de referência de parâmetros bioquímicos e hematológicos dos ratos e ratas Wistar (Rattus novergicus) da UPA/IPeFarM/UFPB. 
Os resultados obtidos para os parâmetros hematológicos estão expressos na Tabela 1, enquanto os dados dos parâmetros bioquímicos estão expressos na Tabela 2.

Tabela 1 - Parâmetros hematológicos de sangue periférico dos ratos e ratas Wistar.

\begin{tabular}{|c|c|c|c|c|}
\hline \multirow{2}{*}{ Parâmetros } & \multicolumn{2}{|c|}{ Ratos } & \multicolumn{2}{|c|}{ Ratas } \\
\hline & Média \pm DP $^{\dagger}$ & Faixa & Média $\pm \mathbf{D P}^{\dagger}$ & Faixa \\
\hline Hemácias $\left(10^{6} / \mu \mathrm{L}\right)$ & $7,49 \pm 0,93$ & $5,63-9,36$ & $7,28 \pm 1,13$ & $5,03-9,54$ \\
\hline RDW (\%) & $11,22 \pm 1,46$ & $8,30-14,14$ & $11,18 \pm 2,15$ & $6,88-15,47$ \\
\hline Hemoglobina (g/dL) & $13,77 \pm 1,86$ & $10,05-17,49$ & $12,93 \pm 2,12$ & $8,70-17,17$ \\
\hline Hematócrito (\%) & $42,74 \pm 5,53$ & $31,68-53,79$ & $40,78 \pm 6,49$ & $27,80-53,76$ \\
\hline $\operatorname{VCM}(\mathbf{f L})$ & $57,12 \pm 2,64$ & $51,83-62,40$ & $56,05 \pm 1,89$ & $52,27-59,83$ \\
\hline HCM (pg) & $18,42 \pm 1,86$ & $14,70-22,13$ & $17,70 \pm 0,94$ & $15,82-19,57$ \\
\hline CHCM (g/dL) & $32,34 \pm 3,41$ & $25,53-39,15$ & $31,66 \pm 1,50$ & $28,66-34,65$ \\
\hline Leucócitos $\left(10^{3} / \mu \mathrm{L}\right)$ & $7,03 \pm 1,26^{\mathrm{a}}$ & $4,52-9,54$ & $5,08 \pm 0,93$ & $3,22-6,94$ \\
\hline Neutrófilos (\%) & $26,57 \pm 4,90$ & $15,77-36,38$ & $27,10 \pm 5,11$ & $16,87-37,33$ \\
\hline Basófilos (\%) & $0,00 \pm 0,00$ & $0,00 \pm 0,00$ & $0,00 \pm 0,00$ & $0,00 \pm 0,00$ \\
\hline Eosinófilos (\%) & $0,00 \pm 0,00$ & $0,00 \pm 0,00$ & $0,00 \pm 0,00$ & $0,00 \pm 0,00$ \\
\hline Linfócitos (\%) & $70,38 \pm 8,12$ & $54,14-86,61$ & $69,38 \pm 7,78$ & $53,83-84,93$ \\
\hline Monócitos (\%) & $3,13 \pm 0,63$ & $1,88-4,39$ & $3,52 \pm 0,51$ & $2,50-4,54$ \\
\hline Plaquetas $\left(10^{3} / \mu \mathrm{L}\right)$ & $798,72 \pm 102,69$ & $593,33-1004,11$ & $742,48 \pm 111,46$ & $519,55-965,41$ \\
\hline VPM (fL) & $5,34 \pm 0,26$ & $4,83-5,86$ & $5,27 \pm 0,40$ & $4,47-6,07$ \\
\hline PDW (\%) & $15,58 \pm 0,38$ & $15,22-16,74$ & $15,87 \pm 0,34$ & $15,20-16,54$ \\
\hline
\end{tabular}

Dados apresentados como média \pm desvio padrão da média e por faixa de valores (mínimo e máximo), analisados por análise de variância (ANOVA) seguido do teste de Tukey. ${ }^{\dagger}$ Desvio padrão. ${ }^{\mathrm{a}} p<0.05$ comparado ao grupo de ratas.

Tabela 2 - Parâmetros bioquímicos dos ratos e ratas Wistar.

\begin{tabular}{ccccc}
\hline \multirow{2}{*}{ Parâmetros } & \multicolumn{2}{c}{ Ratos } & \multicolumn{2}{c}{ Ratas } \\
\cline { 2 - 5 } & ${\text { Média } \pm \mathbf{D P}^{\dagger}}^{\dagger}$ & Faixa & ${\text { Média } \pm \mathbf{D P}^{\dagger}}^{\text {Faixa }}$ \\
\hline Glicose (mg/dL) & $143,71 \pm 12,75$ & $118,21-169,22$ & $155,34 \pm 19,94$ & $115,47-195,21$ \\
Triglicérides (mg/dL) & $80,48 \pm 14,86^{\mathrm{a}}$ & $50,76-110,20$ & $64,97 \pm 12,42$ & $40,12-89,82$ \\
Colesterol (mg/dL) & $60,54 \pm 8,90^{\mathrm{a}}$ & $42,74-78,33$ & $70,68 \pm 12,14$ & $46,41-94,95$ \\
ALT/TGP (U/L) & $15,98 \pm 3,12$ & $9,74-22,23$ & $13,65 \pm 2,45$ & $8,74-18,56$ \\
AST/TGO (U/L) & $51,56 \pm 9,34^{\mathrm{a}}$ & $32,87-70,24$ & $43,14 \pm 6,86$ & $29,42-56,85$ \\
Proteínas totais (g/dL) & $5,95 \pm 0,68$ & $4,59-7,31$ & $6,19 \pm 0,91$ & $4,37-8,02$ \\
Albumina (g/dL) & $2,29 \pm 0,33^{\mathrm{a}}$ & $1,62-2,96$ & $2,70 \pm 0,46$ & $1,77-3,62$ \\
FAL (U/L) & $107,72 \pm 21,54^{\mathrm{a}}$ & $64,95-150,49$ & $57,35 \pm 10,89$ & $35,57-79,12$ \\
Ácido úrico (mg/dL) & $1,13 \pm 0,23^{\mathrm{a}}$ & $0,66-1,60$ & $2,06 \pm 0,36$ & $1,34-2,78$ \\
Ureia (mg/dL) & $39,16 \pm 4,89$ & $29,39-48,93$ & $41,78 \pm 7,90$ & $25,97-57,59$ \\
Creatinina (mg/dL) & $0,42 \pm 0,07$ & $0,28-0,56$ & $0,41 \pm 0,07$ & $0,28-0,54$ \\
\hline
\end{tabular}

Dados apresentados como média \pm desvio padrão da média e por faixa de valores (mínimo e máximo), analisados por análise de variância (ANOVA) seguido do teste de Tukey. 'Desvio padrão. ${ }^{\mathrm{a} p}<0.05$ comparado ao grupo de ratas.

Foram observadas diferenças significativas nos perfis hematológico e bioquímico entre os gêneros masculino e feminino dos animais avaliados. Os ratos apresentaram valores mais elevados para a contagem de leucócitos, a concentração de triglicérides e a atividade enzimática de AST e FAL, quando comparados às ratas; bem como resultados inferiores de ácido úrico, colesterol e albumina, onde as ratas expressaram concentrações superiores para tais analitos.

Uma maior contagem de leucócitos em animais machos pode ser atribuída ao fato de existirem estresse e brigas entre os animais deste gênero, mantidos em uma mesma gaiola durante o desmame, o que faz com que esta população de células se eleve [19]. Dados encontrados, em estudos com 
camundongos, sugerem que machos apresentam valores superiores aos das fêmeas para a contagem total e diferencial de leucócitos [20,21,22]. Tal tendência foi observada no presente trabalho, onde maiores valores de leucócitos foram encontrados nos ratos em relação às ratas.

No que diz respeito aos basófilos e eosinófilos, estas populações raramente são contabilizadas no sangue e pouco diferenciadas entre os granulócitos [23, 24]. Tal característica também foi verificada nesse estudo, no qual houve ausência dessas células nos ratos avaliados.

Outros trabalhos da literatura registraram diferenças significativas nos valores das hemácias, hemoglobina, hematócrito e índices hematimétricos relacionadas com o gênero, para camundongos Swiss [25]; Swiss webster, BALB/c, C57BL/6J e IFN- $\gamma\left({ }^{--}\right)$[24]; e ratos Wistar [26]. No entanto, essas variações não foram observadas na presente pesquisa.

Quanto aos parâmetros bioquímicos, também há relatos na literatura de diferenças entre machos e fêmeas, na avaliação dos analitos do soro/plasma de roedores. Discrepâncias significativas foram verificadas na avaliação de eletrólitos, transaminases, colesterol e glicose entre ratos e ratas Wistar [27]. Estudos com camundongos C57BL/6J verificaram que os machos apresentam concentrações mais elevadas de triglicérides, colesterol, lipoproteína de alta densidade e glicose, em relação às fêmeas [28]. Ainda, foram encontrados valores superiores para atividade enzimática das transaminases e FAL em camundongos machos, em quatro linhagens dessa espécie [24]. No presente trabalho, foram observados valores maiores nos ratos para os triglicérides, AST e FAL, corroborando os dados da literatura. Essa tendência, porém, foi inversa para o colesterol, onde os valores encontrados foram superiores no soro das ratas. Ainda, variações relacionadas com gênero e idade de camundongos para albumina e proteínas totais foram observadas na literatura [25]. Divergências estas que também foram verificadas no presente trabalho, onde as ratas apresentaram resultados superiores na mensuração de albumina.

$\mathrm{Na}$ avaliação dos componentes do sangue, deve-se levar em consideração, também, a influência dos traços fenotípicos, incluindo as variáveis do ambiente, como temperatura, umidade, iluminação, ruído, maravalha, ar, água, tipo de ração e de caixa e quantidade de animais por caixa [21, 29]. Isso porque, em trabalhos que avaliam parâmetros bioquímicos e hematológicos de roedores não tratados, se observa grandes variações nos valores de referência para animais de uma mesma espécie e linhagem, mas com diferentes tipos de acondicionamentos [30, 31, 32, 33]. Por essa razão, torna-se necessário que os biotérios mantenham seu ambiente padronizado, dentro dos limites aceitáveis, conforme preconizado pela Diretriz Brasileira Para o Cuidado e a Utilização de Animais para Fins Científicos, garantindo homogeneidade fisiológica e o bem-estar dos animais [34].

É válido salientar, ainda, que os parâmetros bioquímicos e hematológicos podem ser alterados por diferenças de manuseio e de localização geográfica dos roedores, bem como com a linhagem, gênero e idade de cada espécie estudada $[3,33,35]$. Tais variáveis têm estimulado países com longa tradição em biotérios a determinar e divulgar os parâmetros fisiológicos de seus animais de experimentação, levando em consideração fatores intrínsecos e extrínsecos, a fim de servir de referência para os experimentos de suas instituições de pesquisa [26]. No Brasil, no entanto, poucos dados são encontrados na literatura. Recentemente, a Universidade Federal de Sergipe (UFS) [32], a Universidade Tiradentes (UNIT) [3] e a Universidade Federal do Ceará (UFC) [36] realizaram uma caracterização preliminar das linhagens de animais mantidas em seus biotérios. Tais dados estão expressos nas Tabelas 3 e 4, e foram utilizados para fins comparativos, assim como os valores divulgados pelo laboratório Harlan [37] e pela Charles River [38] (Tabela 5), ambos localizados nos Estados Unidos.

Pode-se observar diferenças nos parâmetros hematimétricos, em que os ratos e ratas da UPA/UFPB expressaram valores inferiores do RDW, em comparação aos ratos e ratas do biotério da UFS; bem como resultados superiores para o VCM, em relação aos animais do biotério da UNIT. Ainda, as ratas da UPA/UFPB apresentaram valores inferiores do CHCM, em relação às ratas do biotério da UNIT, e, quando comparadas às ratas do Harlan, as mesmas expressaram valores inferiores de hemoglobina, hematócrito e HCM.

$\mathrm{Na}$ avaliação da série branca, o número dos leucócitos totais dos animais da UPA/UFPB, em ambos os gêneros, foi superior, em relação aos da Charles River, e inferior, quando comparados aos da UFS e da Harlan. Para ambos os gêneros, ainda, o percentual de neutrófilos dos animais da UPA/UFPB foi superior, em relação aos animais da Harlan e da Charles River. O percentual de linfócitos dos ratos e ratas da UPA/UFPB, por sua vez, foi inferior, em relação aos da Harlan e da 
Charles River. Quanto ao percentual de monócitos, os ratos e ratas da UPA/UFPB apresentaram valores superiores, em relação aos dados da Charles River, e inferiores, quando comparados aos animais da UNIT e da Harlan.

Em relação a série plaquetária, os animais da UPA/UFPB apresentaram valores inferiores no número de plaquetas, frente os ratos e ratas da UNIT, UFS e da Charles River. Ainda, os valores do VPM dos animais da UPA/UFPB, em ambos os gêneros, foram inferiores, quando comparados aos da UFS e da Charles River, assim como os resultados obtidos para PDW, em relação aos dados da Charles River, os quais foram superiores aos da UFPB.

No que diz respeito aos parâmetros bioquímicos, também foram verificadas diferenças nos valores mensurados dos ratos e ratas Wistar da UFPB, quando comparados aos dados da literatura. A glicemia dos animais da UPA/UFPB, em ambos os gêneros, foi superior aos animais da UFS e inferiores aos dados obtidos da UFC; e superiores nas ratas da UPA/UFPB, em relação às ratas da UNIT, Harlan e da Charles River.

Quanto ao perfil lipídico, as concentrações de triglicérides obtidas dos animais da UPA/UFPB, em ambos os gêneros, foram superiores aos da Charles River; e superiores, nos ratos, em relação aos animais da UNIT, assim como as ratas, quando comparadas às ratas do Biotério da UFC. Os valores mensurados para colesterol, por sua vez, foram superiores para as ratas da UPA/UFPB frente os dados da Charles River e do Biotério da UFC; enquanto que, para os ratos, as concentrações desse analito foram inferiores aos animais da Harlan.

No que tange aos parâmetros bioquímicos de dano e função hepática, os resultados obtidos para atividade de ALT e AST dos animais da UPA/UFPB, em ambos os gêneros, foram inferiores aos dados do biotério da UNIT, UFS, Harlan, Charles River e da UFC. A atividade da FAL dos ratos e ratas da UPA/UFPB, por sua vez, foi inferior quando comparada aos animais da Harlan e do Biotério da UFC; e as ratas também expressaram menor atividade dessa enzima, em relação às ratas do biotério da UNIT e da UFS. Quanto à mensuração de albumina, os animais da UPA/UFPB, em ambos os gêneros, apresentaram resultados inferiores aos obtidos para os animais da Harlan, Charles River e do biotério da UFC; e os ratos apresentaram valores inferiores, quando comparados aos ratos da UFS.

Em relação aos parâmetros bioquímicos de função renal, na dosagem de creatinina, os ratos Wistar da UPA/UFPB apresentaram valores superiores, quando comparados aos ratos da Harlan. Para a dosagem de ureia, as concentrações desse analito no soro dos animais da UPA/UFPB foram maiores, em ambos os gêneros, frente os dados da Harlan e Charles River. Em contrapartida, quando confrontados com os dados do biotério da UFC, os ratos da UPA/UFPB expressaram valores inferiores. 
Tabela 3 - Parâmetros hematológicos de ratos e ratas Wistar da UNIT ${ }^{a}$ UFS $^{b}$, Harlan e Charles River.

\begin{tabular}{|c|c|c|c|c|c|c|c|c|}
\hline \multirow{2}{*}{ Parâmetros } & \multicolumn{2}{|c|}{ Biotério da UNIT ${ }^{a}$} & \multicolumn{2}{|c|}{ Biotério da UFS $^{b}$} & \multicolumn{2}{|r|}{ Harlan } & \multicolumn{2}{|c|}{ Charles River } \\
\hline & Ratos & Ratas & Ratos & Ratas & Ratos & Ratas & Ratos & Ratas \\
\hline Hemácias $\left(10^{6} / \mu \mathrm{L}\right)$ & $8,65 \pm 1,11$ & $7,83 \pm 0,69$ & $8,0 \pm 0,55$ & $7,9 \pm 0,34$ & $7,58 \pm 0,35$ & $8,28 \pm 0,46$ & $8,39 \pm 0,67$ & $8,02 \pm 0,53$ \\
\hline RDW (\%) & - & - & $15,90 \pm 1,72$ & $14,79 \pm 1,24$ & - & - & $12,7 \pm 1,1$ & $12,2 \pm 1,2$ \\
\hline Hemoglobina (g/dL) & $15,00 \pm 1,45$ & $14,33 \pm 1,19$ & $14,5 \pm 0,78$ & $14,2 \pm 0,53$ & $14,67 \pm 0,66$ & $16,30 \pm 0,84$ & $15,7 \pm 1,0$ & $15,2 \pm 0,9$ \\
\hline Hematócrito (\%) & $43,30 \pm 3,51$ & $40,52 \pm 3,77$ & $44,2 \pm 2,95$ & $42,9 \pm 2,14$ & $46,15 \pm 2,16$ & $49,73 \pm 2,70$ & $45,0 \pm 3,5$ & $43,3 \pm 3,1$ \\
\hline VCM (fL) & $47,75 \pm 2,89$ & $50,97 \pm 2,00$ & $55,5 \pm 2,22$ & $54,3 \pm 2,80$ & $60,89 \pm 1,62$ & $60,11 \pm 1,05$ & $53,5 \pm 2,4$ & $53,8 \pm 2,3$ \\
\hline HCM (pg) & $16,51 \pm 0,30$ & $18,23 \pm 1,75$ & $18,2 \pm 0,57$ & $17,9 \pm 0,65$ & $19,36 \pm 0,51$ & $19,70 \pm 0,37$ & $18,7 \pm 0,8$ & $19,0 \pm 0,8$ \\
\hline CHCM (g/dL) & $34,89 \pm 2,41$ & $35,76 \pm 3,87$ & $32,8 \pm 1,09$ & $33,1 \pm 1,16$ & $31,79 \pm 0,28$ & $32,78 \pm 0,41$ & $34,9 \pm 1,2$ & $35,3 \pm 1,3$ \\
\hline Leucócitos $\left(10^{3} / \mu \mathrm{L}\right)$ & $7,63 \pm 2,37$ & $4,96 \pm 1,55$ & $9,7 \pm 2,21$ & $8,3 \pm 2,01$ & $12,56 \pm 2,86$ & $11,90 \pm 4,50$ & $4,52 \pm 1,81$ & $3,12 \pm 1,49$ \\
\hline Neutrófilos (\%) & $33,16 \pm 14,99$ & $24,04 \pm 14,55$ & $24,8 \pm 7,85$ & $22,3 \pm 6,7$ & $9,28 \pm 4,45$ & $6,55 \pm 3,47$ & $15,5 \pm 5,5$ & $15,4 \pm 6,4$ \\
\hline Basófilos (\%) & $0,92 \pm 0,74$ & $0,01 \pm 0,03$ & - & - & $0,11 \pm 0,07$ & $0,10 \pm 0,08$ & $0,3 \pm 0,2$ & $0,3 \pm 0,2$ \\
\hline Eosinófilos (\%) & $1,25 \pm 1,09$ & $0,65 \pm 0,61$ & $1,3 \pm 0,82$ & $1,42 \pm 0,75$ & $0,14 \pm 0,07$ & $0,54 \pm 0,26$ & $1,3 \pm 0,8$ & $1,7 \pm 1,0$ \\
\hline Linfócitos (\%) & $67,36 \pm 15,31$ & $73,91 \pm 16,3$ & $70,0 \pm 7,37$ & $71,7 \pm 7,21$ & $81,48 \pm 5,34$ & $86,83 \pm 4,98$ & $80,2 \pm 6,3$ & $80,0 \pm 6,7$ \\
\hline Monócitos (\%) & $5,27 \pm 3,52$ & $5,33 \pm 3,79$ & $3,9 \pm 1,31$ & $3,9 \pm 160$ & $9,01 \pm 2,58$ & $5,99 \pm 2,46$ & $1,9 \pm 0,7$ & $2,0 \pm 0,8$ \\
\hline Plaquetas $\left(10^{3} / \mu \mathrm{L}\right)$ & $982,34 \pm 167,05$ & $971,48 \pm 140,43$ & $1095 \pm 152,56$ & $1004 \pm 150,9$ & - & - & $904 \pm 137$ & $929 \pm 133$ \\
\hline VPM (fL) & - & - & $7,52 \pm 0,35$ & $7,36 \pm 0,28$ & - & - & $7,7 \pm 0,8$ & $7,8 \pm 0,9$ \\
\hline PDW (\%) & - & - & - & - & - & - & $52,9 \pm 5,5$ & $52,7 \pm 5,5$ \\
\hline
\end{tabular}

Dados expressos como média \pm desvio padrão da média. ${ }^{a}$ Universidade Tiradentes; ${ }^{\text {b} U n i v e r s i d a d e ~ F e d e r a l ~ d e ~ S e r g i p e . ~}$ 
Tabela 4 - Parâmetros bioquímicos de ratos e ratas Wistar da UNIT ${ }^{a}$, UFS ${ }^{b}$ e Biotério Central da UFC.

\begin{tabular}{|c|c|c|c|c|c|c|}
\hline \multirow{2}{*}{ Parâmetros } & \multicolumn{2}{|c|}{ Biotério da UNIT ${ }^{a}$} & \multicolumn{2}{|c|}{ Biotério da UFS ${ }^{b}$} & \multicolumn{2}{|c|}{ Biotério Central da UFC } \\
\hline & Ratos & Ratas & Ratos & Ratas & Ratos & Ratas \\
\hline Glicose (mg/dL) & $138,72 \pm 30,17$ & $114,57 \pm 36,15$ & $104,0 \pm 17,18$ & $106,2 \pm 20,25$ & $187,35 \pm 38,0$ & $201,38 \pm 27,43$ \\
\hline Triglicérides (mg/dL) & $46,87 \pm 18,73$ & $54,21 \pm 35,5$ & $89,9 \pm 29,16$ & $69,8 \pm 20,75$ & $60,35 \pm 11,86$ & $51,57 \pm 8,08$ \\
\hline Colesterol (mg/dL) & $60,68 \pm 6,51$ & $64,86 \pm 11,17$ & $67,4 \pm 8,72$ & $75,1 \pm 12,29$ & $62,95 \pm 14,23$ & $55,86 \pm 10,53$ \\
\hline ALT/TGP (U/L) & $57,55 \pm 11,95$ & $45,47 \pm 9,23$ & $48,4 \pm 6,46$ & $41,0 \pm 7,63$ & $54,95 \pm 9,89$ & $31,95 \pm 8,59$ \\
\hline AST/TGO (U/L) & $131,33 \pm 43,98$ & $107,87 \pm 53,96$ & $131,7 \pm 23,09$ & $132,7 \pm 27,23$ & $104,35 \pm 12,8$ & $109,10 \pm 13,07$ \\
\hline Proteínas totais (g/dL) & $5,75 \pm 0,87$ & $6,07 \pm 0,83$ & $6,2 \pm 0,26$ & $6,5 \pm 0,29$ & $5,81 \pm 0,56$ & $5,78 \pm 0,22$ \\
\hline Albumina (g/dL) & $2,65 \pm 0,30$ & $2,41 \pm 0,76$ & $3,0 \pm 0,12$ & $3,1 \pm 0,16$ & $4,68 \pm 0,54$ & $4,79 \pm 0,19$ \\
\hline FAL $(\mathbf{U} / \mathbf{L})$ & $91,63 \pm 28,70$ & $75,95 \pm 19,07$ & $127,1 \pm 35,55$ & $106,9 \pm 20,69$ & $368,75 \pm 33,9$ & $250,10 \pm 36,50$ \\
\hline Ácido úrico (mg/dL) & $1,81 \pm 0,67$ & $1,82 \pm 0,59$ & $1,3 \pm 0,34$ & $1,6 \pm 0,39$ & - & - \\
\hline Ureia (mg/dL) & $39,97 \pm 6,78$ & $39,17 \pm 6,43$ & $35,9 \pm 3,58$ & $43,7 \pm 7,42$ & $53,15 \pm 11,77$ & $49,76 \pm 6,01$ \\
\hline Creatinina (mg/dL) & $0,58 \pm 0,24$ & $0,57 \pm 0,19$ & $0,5 \pm 0,05$ & $0,5 \pm 0,07$ & $0,33 \pm 0,15$ & $0,47 \pm 0,15$ \\
\hline
\end{tabular}

Dados expressos como média \pm desvio padrão da média. ${ }^{a}$ Universidade Tiradentes; ${ }^{b}$ Universidade Federal de Sergipe; ${ }^{c}$ Universidade Federal do Ceará.

Tabela 5 - Parâmetros bioquímicos de ratos e ratas Wistar da Harlan e Charles River.

\begin{tabular}{|c|c|c|c|c|}
\hline \multirow{2}{*}{ Parâmetros } & \multicolumn{2}{|c|}{ Harlan } & \multicolumn{2}{|c|}{ Charles River } \\
\hline & Ratos & Ratas & Ratos & Ratas \\
\hline Glicose (mg/dL) & $159,55 \pm 23,18$ & $121,66 \pm 42,41$ & $123,0 \pm 38,0$ & $117,0 \pm 25,0$ \\
\hline Triglicérides (mg/dL) & - & - & $44,0 \pm 21,0$ & $28,0 \pm 8,0$ \\
\hline Colesterol (mg/dL) & $95,19 \pm 12,67$ & $78,11 \pm 20,12$ & $58,0 \pm 13,0$ & $48,0 \pm 13,0$ \\
\hline ALT/TGP (U/L) & $76,13 \pm 11,36$ & $64,28 \pm 10,23$ & $28,0 \pm 7,0$ & $25 \pm 9,0$ \\
\hline AST/TGO (U/L) & $131,03 \pm 44,00$ & $105,66 \pm 23,01$ & $105,0 \pm 20,0$ & $102,0 \pm 31,0$ \\
\hline Proteínas totais (g/dL) & $5,89 \pm 0,16$ & $6,28 \pm 0,27$ & $6,0 \pm 0,5$ & $6,3 \pm 0,5$ \\
\hline Albumina (g/dL) & $2,91 \pm 0,14$ & $3,31 \pm 0,24$ & $4,0 \pm 0,4$ & $4,4 \pm 0,5$ \\
\hline FAL $(\mathbf{U} / \mathbf{L})$ & $237,06 \pm 33,93$ & $112,46 \pm 28,11$ & $113,0 \pm 44,0$ & $59,0 \pm 28,0$ \\
\hline Ácido Úrico (mg/dL) & - & - & - & - \\
\hline Ureia (mg/dL) & $12,73 \pm 1,83$ & $13,66 \pm 2,14$ & $17,1 \pm 2,9$ & $19,3 \pm 3,7$ \\
\hline Creatinina (mg/dL) & $0,23 \pm 0,03$ & $0,33 \pm 0,04$ & $0,3 \pm 0,1$ & $0,4 \pm 0,1$ \\
\hline
\end{tabular}

Dados expressos como média \pm desvio padrão da média. 
As diferenças observadas nos resultados do presente estudo frente os dados da literatura podem ser indicativos de variações intraespécies nos animais, decorrentes da influência do meio. Contudo, é importante salientar que, em análises clínicas, algumas variáveis podem interferir nos resultados obtidos para uma amostra biológica, incluindo fatores pré-analíticos (jejum, tipo de coleta, condições de manuseio e a preservação dos analitos) [39, 40, 41]; analíticos (processamento da amostra, método empregado, equipamentos, reagentes, calibradores, tipo de dados brutos e método do cálculo) [42, 43]; e pós-analíticos (geração do resultado à emissão do laudo) [44, 45, 46]. Com efeito, diferenças nos aspectos pré-analíticos e analíticos foram observadas entre os trabalhos da literatura e a presente pesquisa. O estudo da UFC, por exemplo, diferiu desse trabalho quanto ao número de animais (19 animais/gênero), faixa etária (6 a 8 semanas), via de coleta do sangue (aorta abdominal), jejum (não aplicado), e os equipamentos utilizados nas análises bioquímicas e hematológicas (automatizados).

De maneira semelhante, a pesquisa realizada pela UFS diferiu do presente estudo, ao passo que empregou uma quantidade de animais variável, de 11 a 41 ratos para cada parâmetro, anestesia por inalação de éter e equipamentos automatizados. Em adição, a empresa Charles River realizou a padronização dos parâmetros bioquímicos e hematológicos reunindo-se dados laboratoriais dos grupos controles de 30 estudos (de 2002 a 2007) conduzidos com os ratos Wistar, na faixa etária de 8 a 16 semanas. A coleta de sangue, nesse estudo, foi realizada pela aorta abdominal, após jejum (tempo não informado) e os parâmetros foram avaliados com metodologias automatizadas, onde $o$ número de animais para os parâmetros hematológicos variou de 174 a 181 ratos/gênero e para os bioquímicos de 158 a 165 animais. Já em relação aos dados fornecidos pela Harlan, os detalhes técnicos não foram descritos, sendo informado apenas o número de animais, que foi de 40 ratos/gênero, e a idade, que foi de 7 a 8 semanas.

Valores discrepantes podem ser verificados devido aos diferentes métodos utilizados para os testes enzimáticos, de modo que a comparação entre estudos, ainda que com a mesma unidade de medida, só é válida se forem utilizados os mesmos substratos, $\mathrm{pH}$ e temperatura de reação [47]. Estudos revelam que a metodologia empregada sempre contribui com os resultados laboratoriais [48] e a generalização dos dados só é possível em determinadas condições específicas de estudo, com a mesma linhagem de ratos e metodologia [49].

Vários trabalhos da literatura sugerem, ainda, que o tipo de anestésico, a idade, a via de coleta e o tempo de jejum podem causar diferenças significativas nos valores obtidos para parâmetros bioquímicos e hematológicos de animais de laboratório [20, 50, 51, 52, 53]. Com efeito, tais variáveis devem ser as mais semelhantes possíveis entre os trabalhos que analisam tais parâmetros, para uma comparação mais coerente dos dados, evitando conclusões equivocadas [50, 54]. Logo, as divergências metodológicas encontradas na padronização das UFS, UFC, Charles River, Harlan, em relação ao presente estudo, podem ser responsáveis pelas diferenças entre os resultados obtidos para os parâmetros bioquímicos e hematológicos de tais instituições, o que inviabiliza uma comparação exata dos dados.

$\mathrm{O}$ trabalho que mais se aproximou, metodologicamente, dessa pesquisa foi o da padronização realizada no biotério da Universidade Tiradentes, onde foram empregados 44 animais/gênero/linhagem, com faixa etária de 8 a 12 semanas, os quais foram submetidos ao jejum de 12 horas, anestesiados com cetamina e xilazina e o sangue foi coletado por punção intracardíaca. A semelhança técnica encontrada entre o presente estudo e o da UNIT sugeria que os valores de referência dos animais de ambos os trabalhos seriam semelhantes [3, 32]. Em contrapartida, isso não foi observado quando se comparou os dados dos referidos estudos, sendo verificados concentrações mais elevadas de glicose, triglicérides, VCM e da atividade enzimática da FAL dos animais da UPA/UFPB, em relação aos animais da UNIT, bem como resultados inferiores de monócitos, plaquetas, CHCM e atividade enzimática da ALT e AST, respectivamente. Tais variações nos animais de mesma espécie e linhagem, mas de criadouros diferentes, corroboram a necessidade de se determinar os valores de referência específicos para cada biotério, considerando diferenças de ordem ambiental, manuseio e/ou metodologia empregada [3, 32, 33].

É importante salientar, contudo, que uma comparação exata entre o presente trabalho e o da UNIT só seria possível avaliando-se os dados brutos de ambas pesquisas, no intuito de verificar a significância das diferenças apontadas. Ademais, é preciso considerar que o fenótipo hematológico e bioquímico dos roedores ainda é pouco conhecido e uma análise detalhada dos dados seria 
necessária para apontar as reais causas dessas diferenças. Algumas variáveis específicas, contudo, têm sido apontadas, na literatura, como importantes na fisiologia dos roedores, incluindo o próprio genótipo e a ninhada de origem desses animais, além de fatores extrínsecos, relativos ao meio [24, 55]. Destes, o tipo de alimentação e de maravalha, a interação do roedor com o experimentador, a técnica de manuseio, o período do dia em que é realizada a coleta do sangue, bem como o estresse animal durante o experimento podem influenciar, significativamente, os dados referentes ao perfil fisiológico dos animais [31, 32, 33, 56]. Assim, pode-se sugerir que variáveis genéticas e ambientais podem estar relacionadas com as discrepâncias nos parâmetros bioquímicos e hematológicos encontradas entre os ratos da UPA/UFPB e da UNIT.

Outros trabalhos também verificaram divergências significativas nos perfis bioquímicos e hematológicos em animais de acordo com as condições ambientais. Há relatos, por exemplo, de diferenças nos valores de referência na mensuração de colesterol, triglicérides, glicose, eletrólitos e atividade enzimática da AST e ALT, em camundongos C57BL/6J e BALB/c criados em diferentes condições sanitárias [14]. Ainda, trabalhos realizados no antigo Laboratório de Tecnologia Farmacêutica-UFPB verificaram alterações nos parâmetros bioquímicos e hematológicos de camundongos Swiss e ratos Wistar, após mudanças nos critérios de acondicionamento desses animais [7, 26]. De maneira semelhante, parâmetros bioquímicos e hematológicos variaram em camundongos Swiss webster, BALB/c, C57BL/6J e IFN- $\gamma(-/-)$ mantidos em condições diferentes, nos criadouros da Fundação Oswaldo Cruz [24].

A interação entre o genótipo e os aspectos ambientais é reconhecida como uma importante fonte de variações experimentais, quando características complexas são mensuradas em roedores [56]. Vale salientar que tais divergências de dados podem ser verificadas mesmo entre animais mantidos em condições ambientais idênticas, mas originados de diferentes biotérios. Como exemplo, discrepâncias relevantes nos valores de leucócitos, eritrócitos, plaquetas, linfócitos, monócitos, eosinófilos, reticulócitos, VCM, HCM, hematócrito e hemoglobina foram observadas em estudo com ratos Wistar obtidos de dois criadouros distintos [55]. É importante salientar que esses ratos foram mantidos em aclimatação idêntica, em mesmo ambiente, durante 3 a 5 semanas, antes da coleta de sangue, sendo a origem dos mesmos a única diferença entre os grupos. Assim, animais de mesma linhagem, com mesmo acondicionamento, podem expressar diferenças em seus perfis fisiológicos devido a interação com o meio de origem. Tal interação pode levar a alterações genéticas e epigenéticas em genes responsáveis pela hematopoese e pelas características bioquímicas dos animais, ocasionando, portanto, as divergências em sua fisiologia. Uma análise genética detalhada seria útil para compreender essa variabilidade hematológica e bioquímica entre os animais de mesma espécie e linhagem [24, 55].

Portanto, considerando a influência do genótipo e do meio na fisiologia do animal, torna-se evidente a necessidade de cada instituição de pesquisa estabelecer seus próprios intervalos de referência dos parâmetros bioquímicos e hematológicos dos animais de laboratório, em vez de utilizar dados de outros criadouros. Ainda, conforme recomendado pelo Joint Scientific Committee for International Harmonization of Clinical Pathology Studies, os intervalos de referência devem ser constantemente revisados sempre que houver mudanças de fornecedor de animais e nos critérios de acondicionamento dos mesmos [57].

\section{CONCLUSÃO}

Os resultados obtidos nesse estudo são dados preliminares de uma caracterização de parâmetros bioquímicos e hematológicos dos ratos e ratas Wistar, provenientes da Unidade de Produção Animal do Instituto de Pesquisa em Fármacos e Medicamentos da Universidade Federal da Paraíba. Tais parâmetros são essenciais para a avaliação da homeostase, compreensão dos processos vitais e patológicos, e comparação dos grupos tratados com amostras em estudo e grupos sadios dos animais empregados para fins experimentais nessa instituição.

Ainda, as análises comparativas realizadas nesse estudo demonstraram que animais de mesma espécie e linhagem podem expressar valores divergentes em seus perfis bioquímico e hematológico, associados com o gênero e biotério/criadouro de origem, o que pode estar relacionado a fatores ambientais, genéticos e epigenéticos. Assim, esse estudo corrobora a necessidade do 
estabelecimento de valores de referência para cada biotério, considerando gênero, linhagem e os aspectos metodológicos, que devem ser bem padronizados.

É importante frisar que novas determinações de parâmetros fisiológicos devem ser realizadas, cada vez que os animais são mantidos em condições experimentais particulares. No entanto, vale ressaltar que essas divergências significativas nos perfis bioquímico e hematológico de animais obtidos de diferentes unidades de produção devem ser minimizadas com a implementação de normas padronizadas pelos órgãos regulatórios, no que se refere a aclimatação e abordagens metodológicas, com o objetivo de maximizar a reprodutibilidade dos resultados experimentais encontrados nos diferentes centros de pesquisa.

\section{AGRADECIMENTOS}

Os autores agradecem aos funcionários da UPA/IPeFarM, a empresa Bioclin ${ }^{\circledR}$ pela doação dos kits para as análises bioquímicas e o apoio institucional da UFPB.

\section{REFERÊNCIAS BIBLIOGRÁFICAS}

1. Almeida RN. Psicofarmacologia: fundamentos práticos. Rio de Janeiro: Guanabara Koogan; 2006. 357 p.

2. Raymundo MM, Goldim JR. Ética da pesquisa em modelos animais. Rev Bio. 2009;10(1):31-44.

3. Lima CM, Lima AK, Melo MGD, Dória GAA, Serafini MR, Albuquerque-Júnior RLC, Araújo AAS. Valores de referência hematológicos e bioquímicos de ratos (Rattus novergicus linhagem Wistar) provenientes do biotério da Universidade Tiradentes. Sci Plena. 2014 Mar;10(3):1-9.

4. Guénet JL. Animal models of human genetic diseases: do they need to be faithful to be useful? Mol Genet Genom. 2011 Jul;286(1):1-20, doi:10.1007/s00438-011-0627-y.

5. Mendes MMPG, De Jesus Souza CSA. Aplicação de modelos animais na pesquisa biomédica experimental. RSF. 2017 Dez;4(2):41-58.

6. Chorilli M, Michelin DC, Salgado, HRN. Animais de laboratório: o camundongo. J Basic Appl Pharm Sci. 2007 Jul;28(1):1-23.

7. Branco ACS, Diniz MFFM, Almeida RN, Santos HB, Oliveira KM, Ramalho JÁ, Dantas JG. Parâmetros bioquímicos e hematológicos de ratos Wistar e camundongos Swiss do biotério professor Thomas George. Rev Bras Ciênc Saúde. 2011 Fev;15(2):209-214, doi:10.4034/RBCS.2011.15.02.11.

8. Andrade A, Pinto SC, Oliveira RS. Animais de laboratório: criação e experimentação. Rio de Janeiro: Editora Fiocruz; 2002. 388 p.

9. Magalhães, LE. A ciência e os animais de laboratório. RESBCAL. 2012 Mar;1(1):7-13.

10. Madureira KM, Gomes V, Barcelos B, Zani BH, de Lara Shecaira C, Baccili CC, Benesi FJ. Parâmetros hematológicos e bioquímicos de ovinos da raça Dorper. Semin Cienc Agrar. 2013 Abr;34(2):811-816, doi: 10.5433/1679-0359.2013v34n2p811.

11. Lapchik VBV, Mattaraia VGM, Ko GM. Cuidados e Manejo de Animais de Laboratório. $2^{\mathrm{a}}$ ed. São Paulo: Atheneu; 2010. 708 p.

12. Harkness JE, Wagner JE. Biologia e clínica de coelhos e roedores. $3^{\text {a }}$ ed. São Paulo: Livraria Roca Ltda; 1993. $238 \mathrm{p}$.

13. Olfertt ED, Cross BM, McWilliam AA. Guide to the care and use of experimental animals. Ottawa (CA): Canadian Council on Animal Care, 1993. 299 p.

14. Spinelli MO, Cruz RJ, Godoy CMS, Motta MC. Comparação dos parâmetros bioquímicos de camundongos criados em diferentes condições sanitárias. Sci Plena. 2012 Jan;8(2):1-8.

15. Shahsavani D, Kazerani HR, Kaveh S, Gholipour-Kanani H. Determination of some normal serum parameters in starry sturgeon (Acipenser stellatus Pallas, 1771) during spring season. Comp Clin Path. 2010 Feb;19(1):57-61, doi: 10.1007/s00580-009-0899-3.

16. Pérez JM, González FJ, Granados JE, Pérez MC, Fandos P, Soriguer RC, Serrano E. Hematologic and biochemical reference intervals for Spanish ibex. J Wildl Dis. 2003 Jan;39(1):209-215, doi: 10.7589/0090-3558-39.1.209.

17. Horowitz GL. Reference intervals: practical aspects. EJIFCC. 2008 Oct;19(2):95-105.

18. Friedrichs KR, Harr KE, Freeman KP, Szladovits B, Walton RM, Barnhart KF, Blanco-Chavez J. ASVCP reference interval guidelines: determination of de novoreference intervals in veterinary species and other related topics. Vet Clin Pathol. 2012 Dec;41(4):441-453, doi:10.1111/vcp.12006.

19. Kile BT, Mason-Garrison CL, Justice MJ. Sex and strain-related differences in the peripheral blood cell values of inbred mouse strains. Mamm Genome. 2003 Jan;14(1):81-85, doi:10.1007/s00335-002-2160-0. 
20. Schnell MA, Hardy C, Hawley M, Propert KJ, Wilson JM. Effect of blood collection technique in mice on clinical pathology parameters. Hum Gene Ther. 2002 Jan;13(1):155-161, doi: $10.1089 / 10430340152712700$.

21. Mazzaccara C, Labruna G, Cito G, Scarfò M, De Felice M, Pastore L, Sacchetti L. Age-related reference intervals of the main biochemical and hematological parameters in C57BL/6J, 129SV/EV and C3H/HeJ mouse strains. PloS one. 2008 Nov;3(11):e3772, doi: 10.1371/journal.pone.0003772.

22. Wirth-Dzięciołowska E, Karaszewska J, Pyśniak K, Smolińska M, Gajewska M. Selected peripheral blood cell parameters in twelve inbred strains of laboratory mice. Anim Sci Pap Rep. 2009 Dec;27(1):6977.

23. Dvorak AM. The mouse basophil, a rare and rarely recognized granulocyte. Blood. 2000 Aug;96(4):16161617, doi: 10.1182/blood.V96.4.1616.

24. Araújo FTM. Estabelecimento de valores de referência para parâmetros hematológicos e bioquímicos e avaliação do perfil imunológico de linhagens de camundongos produzidas nos Biotérios do Centro de Pesquisas René Rachou/FIOCRUZ - Minas e do Centro de Criação de Animais de Laboratório/FIOCRUZ [dissertação]. Belo Horizonte (MG): Fundação Oswaldo Cruz; 2012. 157 p.

25. Restel TI. Avaliação ponderal, hematólogica e bioquímica em camundongos Mus musculus, linhagem Swiss, machos e fêmeas de diferentes idades, do biotério central da Universidade Federal de Mato Grosso do Sul, Campo Grande, MS [dissertação]. Campo Grande (MS): Universidade Federal de Mato Grosso do Sul; 2012. $72 \mathrm{p}$.

26. Diniz MFFM, Medeiros IA, Santos HB, Oliveira KM, Vasconcelos THC, Aguiar FB, Toscano MG, Ribeiro ÊAN. Padronização do parâmetros hematológicos e bioquímicos de camundongos Swiss e ratos Wistar. Rev Bras de Saúde. 2006;10(2):171-176.

27. Boehm O, Zur B, Koch A, Tran N, Freyenhagen R, Hartmann M, Zacharowski K. Clinical chemistry reference database for Wistar rats and C57/BL6 mice. Biol Chem. 2007 Nov;388(11):1255-1256, doi: 10.1515/BC.2007.061.

28. Zhou X, Hansson GK. Effect of sex and age on serum biochemical reference ranges in C57BL/6J mice. Comp Med. 2004 Apr;54(2):176-178.

29. Lapchik VBV, Mattaraia VGM, K GM. Cuidados e manejo de animais de laboratório. São Paulo: Atheneu; 2010. $615 \mathrm{p}$.

30. Bruno GB, Menezes VA, Bruno JA, Almeida MW, Viana GSB. Avaliações hematológicas e bioquímicas do sangue de cães submetidos à pulpotomias com cimento de antibiótico. Rev Odontol UNESP. 2016;35(3):125-133.

31. Carvalho GD, Masseno APB, Zanini MS, Zanini SF, Porfírio LC, Machado JP, Mauad H. Avaliação clínica de ratos de laboratório (Rattus novergicus linhagem Wistar): parâmetros sanitários, biológicos e fisiológicos. Rev Ceres. 2009 Out;56(1):51-57.

32. Melo MGD, Dória GAA, Serafini MR, Araújo AAS. Valores de referência hematológicos e bioquímicos de ratos (Rattus novergicus linhagem Wistar) provenientes do biotério central da Universidade Federal de Sergipe. Sci Plena. 2012 Abr;8(4):171-176.

33. Dantas JA, Ambiel CR, Cuman RKN, Baroni S, Bersani-Amado CA. Valores de referência de alguns parâmetros fisiológicos de ratos do Biotério Central da Universidade Estadual de Maringá, Estado do Paraná. Acta Sci, Health Sci. 2006;28(2):165-170, doi: 10.4025/actascihealthsci.v28i2.1099.

34. Conselho Nacional de Controle e Experimentação Animal (CONCEA). Diretriz brasileira para o cuidado e a utilização de animais para fins científicos. Brasília, 2013. 50 p.

35. Damy SB, Camargo RS, Chammas R, Figueiredo LFPD. Aspectos fundamentais da experimentação animal-aplicações em cirurgia experimental. Rev Assoc Med Bras. 2010;56(1):103-111.

36. Lima FEG, Santos MSN, Bachur TPR, Moraes Filho MO, Moraes MEA, Aragão GF. Perfil bioquímico de ratos Wistar do Biotério Central da Universidade Federal do Ceará. Ci. Anim. 2018;28(3):1-11.

37. Harlan Laboratories. Wistar, Hsd:WI. [Internet]. Indianapolis (NO): Harlan Laboratories; 2014. Disponível em: https://www.envigo.com/model/hsd-wi

38. Charles Rivers Laboratories. Clinical laboratory parameters for Crl:CD (SD) BR. Wilmington (DE): Charles Rivers Laboratories; 2008. 14 p.

39. Vieira JGH. Avaliação dos potenciais problemas pré-analíticos e metodológicos em dosagens hormonais. Arq Bras Endocrinol Metabol. 2002 Fev;46(1):9-15, doi: 10.1590/S0004-27302002000100003.

40. Lima-Oliveira GS, Picheth G, Sumita NM, Scartezini M. Controle da qualidade na coleta do espécime diagnóstico sanguíneo: iluminando uma fase escura de erros pré-analíticos. J Bras Patol Med Lab. 2009 Dez;45(6):441-447, doi: 10.1590/S1676-24442009000600002.

41. Lima-Oliveira G. Gestão da qualidade laboratorial: é preciso entender as variáveis para controlar o processo e garantir a segurança do paciente. Análises Clínicas. 2011;1(1):1-12.

42. International Organization for Standardization. ISO/TS 11405:2015: dentistry: testing of adhesion to tooth structure. Geneva (CH): ISO; 2015. 12 p. 
43. Burtis CA, Ashwood Bruns DE. Tietz. Fundamentos de química clínica. Rio de Janeiro: Elsevier; 2008. $959 \mathrm{p}$.

44. Harris EK, Yasaka T. On the calculation of a" reference change" for comparing two consecutive measurements. Clin Chem. 1983 Jan;29(1):25-30, doi: 10.1093/clinchem/29.1.25.

45. Minitério da Saúde, Agência Nacional de Vigilância Sanitária. Resolução № 302, de 13 de outubro de 2005. Dispõe sobre Regulamento Técnico para funcionamento de Laboratórios Clínicos. Diário Oficial da União. 14 out 2005;198(Seção 1):33.

46. Weiss DJ, Wardrop KJ. Schalm's Veterinary Hematology. Ames (IA): Wiley Blackwell; 2010. 1206 p.

47. Duncan JR, Prasse KW. Patologia Clínica Veterinária, Apêndice I. Rio de Janeiro: Guanabara Koogan; $1982,217 \mathrm{p}$.

48. Haldeman CT, Blue J. Veterinary laboratory medicine: in pratice, Treton (NJ): Veterinary Learning Systems; 1993. $84 \mathrm{p}$.

49. Lillie LE, Temple NJ, Florence LZ. Reference values for young normal Sprague-Dawley rats: weight gain, hematology and clinical chemistry. Hum Exp Toxicol. 1996 Aug;15(8):612-616, doi: $10.1177 / 096032719601500802$.

50. Fernández I, Peña A, Del Teso N, Pérez V, Rodrígues-Cuesta J. Clinical biochemistry parameters in C57BL/6J mice after blood collection from the submandibular vein and retroorbital plexus. J Am Assoc Anim Sci. 2010 Mar;49(2):202-206.

51. Bernardi C, Monetal D, Brughera M, Di Salvo M, Lamparelli D, Mazue G, Iatropoulos MJ. Haematology and clinical chemistry in rats: comparison of different blood collection sites. Comp Haematol Int. 1996 Sep;6(3):160-166.

52. Gonzalez Gil A, Illera JC, Silvan G, Illera M. Effects of the anaesthetic/tranquillizer treatments on selected plasma biochemical parameters in NZW rabbits. Lab Anim. 2003 Apr;37(2):155-161, doi: $10.1258 / 00236770360563804$.

53. Fukuda S, Tsuchikura S, Iida H. Age-related changes in blood pressure, hematological values, concentrations of serum biochemical constituents and weights of organs in the SHR/Izm, SHRSP/Izm and WKY/Izm. Exp Anim. 2004 Sep;53(1):67-72, doi: 10.1538/expanim.53.67.

54. Matida ET, Zancanaro AE, Restel TI, Gomes VMW, Bazzano T, Mori CMC, Teixeira MA. Determinação de parâmetros bioquímicos e hematológicos em camundongos (Mus musculus) do biotério central da UFMS. RESBCAL. 2015 Mar;3(1):30-35.

55. Kampfmann I, Bauer N, Johannes S, Moritz A. Differences in hematologic variables in rats of the same strain but different origin. Vet Clin Pathol. 2012 May;41(2):228-234, doi: 10.1111/j.1939165X.2012.00427.x.

56. Valdar W, Solberg LC, Gauguier D, Cookson WO, Rawlins JNP, Mott R, Flint J. Genetic and environmental effects on complex traits in mice. Genetics. 2006 Out;174(2):959-984, doi: 10.1534/genetics.106.060004.

57. Weingand K, Brown G, Hall R, Davies D, Gossett K, Neptun D, Waner T, Matsuzawa T, Salemink P, Froelke W, Provost JP, Dal Negro G, Batchelor J, Nomura M, Groetsch H, Boink A, Kimball J, Woodman D, York M, Fabianson-Johnson E, Lupart M, Melloni E. Harmonization of animal clinical pathology testing in toxicity and safety studies. The Joint Scientific Committee for International Harmonization of Clinical Pathology Testing. Fundam Appl Toxicol. 1996 Feb;29(2):198-201. 